and 23 genes had a higher expression level in responders. Figure 1 showed the top 20 DEG Heatmap between the non-responder and responders.

Using these two sets of genes for GO analysis, we found that most of the pathways in the non-responder are related to immune response and cytokine production, and most of the pathways in the responders are related to antigen processing and $\mathrm{MHC}$ class II

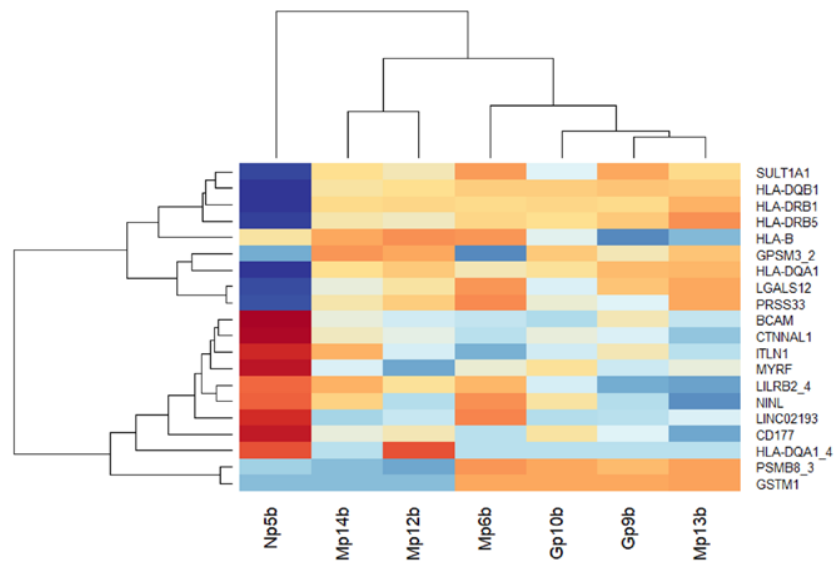

Figure 1. Top 20 DEG Heatmap between non-responder and responders

Conclusion: The study showed that most of the pathways in RA patients with no EULAR response to abatacept are related to immune response and cytokine production; while most of the pathways in RA patients with moderate/good response to abatacept are related to antigen processing and $\mathrm{MHC}$ class II. REFERENCES:

[1] Yokoyama-Kokuyo W, Yamazaki H, Takeuchi T, et al. Identification of molecules associated with response to abatacept in patients with rheumatoid arthritis. Arthritis Research \& Therapy. 2020;22:46.

Disclosure of Interests: Hsin-Hua Chen Grant/research support from: This is an investigator-sponsored trial with Bristol-Myers Squibb who provides funding support., Wen-Cheng Chao: None declared, Jing-Rong Wang: None declared, Tai-Ming Ko: None declared

DOI: 10.1136/annrheumdis-2021-eular.896

\section{AB0109 VALIDATION OF SEPARATE PATIENT-REPORTED, CLINICAL AND LABORATORY FACTOR SCORES AS REPRESENTATION OF DISEASE BURDEN IN A POPULATION WITH ESTABLISHED RHEUMATOID ARTHRITIS}

S. Pazmino ${ }^{1}$, A. Lovik ${ }^{2}$, D. De Cock ${ }^{1}$, V. Stouten ${ }^{1}$, D. Bertrand ${ }^{1}$, J. Joly ${ }^{3}$, M. Doumen ${ }^{1}$, R. Westhovens ${ }^{1,3}$, P. Verschueren ${ }^{1,3}$ on behalf of TapERA study group. ${ }^{1}$ Katholieke Universiteit Leuven, Skeletal Biology and Engineering Research Centre, Department of Development and Regeneration, Leuven, Belgium; ${ }^{2}$ Katholieke Universiteit Leuven, I-Biostat, Leuven, Belgium; ${ }^{3}$ UZ Gasthuisberg Campus, Department of Rheumatology, Leuven, Belgium

Background: Rheumatoid arthritis (RA) can cause important bio-psychosocial burden. When exploring disease burden evolution in the 2-year Care in early RA (CareRA) trial, 3 factor scores were extracted via exploratory factor analysis (EFA). ${ }^{1}$ EFA uncovers the fact that multiple observed variables have similar patterns of responses because they are all associated with a latent, not directly observable, variable.

Objectives: To validate in a population with established RA, the 3 factors scores and their individual components originally extracted in CareRA.

Methods: Patients with established RA in sustained remission under treatment with etanercept ( $\geq 1$ year) were enrolled in the TapERA (Tapering Etanercept in RA) trial between 2012 and 2014. Patients completed the Flare Assessment in RA (FLARE-RA) questionnaire. ${ }^{2}$

Components of disease activity scores (swollen/tender joint count, physician and patient global health assessment, CRP and ESR), as well as pain (question 2) and fatigue evaluation (question 8), from the FLARE-RA questionnaire, and HAQ were recorded at every visit $(\mathrm{v}=5)$.

Missingness on previously mentioned variables was handled with multiple imputation (100 imputations). Pain and fatigue were re-scaled from their original Likert scale of 1-6 to 0-100 to match CareRA data. Next, timepoint clustering was removed with multiple outputation (1000x) and each of the 100000 datasets was analyzed by EFA with principal component extraction and oblimin rotation. The analyses were combined after re-ordering the factors by maximizing factor congruence.

Results: Sixty-six patients with a mean disease duration of 14.8 years (SD 9.03), mean age of 55.21 years (SD12.87), $96 \%$ (63/66) positive to RF or ACPA, $77 \%$ $(51 / 66)$ with erosions and $68 \%(45 / 66)$ female were included in this analysis. Table 1 provides the results of the EFAs from CareRA and TapERA. The factor structure and factor components remained the same in both datasets. The factor loadings, indicating how strongly a variable relates to its factor (correlation between observed and latent score), were also comparable. The HAQ, however; did have a stronger factor loading in TapERA (0.57 vs 0.92).

Table 1. Results from the exploratory factor analyses in CareRA and TapERA

\begin{tabular}{|c|c|c|c|c|c|c|}
\hline \multirow[t]{2}{*}{ Variable } & \multicolumn{3}{|c|}{ CareRA } & \multicolumn{3}{|c|}{ TapERA } \\
\hline & PRF & CF & LF & PRF & $\mathrm{CF}$ & LF \\
\hline Fatigue & 0.90 & & & 0.80 & & \\
\hline PaGH & 0.87 & & & 0.81 & & \\
\hline Pain & 0.86 & & & 0.75 & & \\
\hline HAQ & 0.57 & & & 0.92 & & \\
\hline SJC28 & & 0.92 & & & 0.82 & \\
\hline TJC28 & & 0.89 & & & 0.84 & \\
\hline PhGH & & 0.76 & & & 0.60 & \\
\hline CRP & & & 0.87 & & & 0.85 \\
\hline ESR & & & 0.78 & & & 0.82 \\
\hline
\end{tabular}

Factor loadings presented (correlation between the observed score and the latent factor). Cross-loadings were negligible $(<0.3)$-not presented. The factor order is by $\%$ of variance explained.PRF: patient-reported factor, CF: clinical factor, LF: laboratory factor, $\mathrm{PaGH}$ patient's global health assessment, HAQ: health assessment questionnaire, SJC28: 28 swollen joint count, TJC28: 28 tender joint count, PhGH: physician's global health assessment, CRP: C-reactive protein, ESR: erythrocyte sedimentation rate

Conclusion: The latent factor structure for disease burden originally found in CareRA was successfully validated in the TapERA dataset, underlining the robustness of the PRF, CF and LF scores. HAQ seems to take "greater importance" on established RA. However, deviations in factor loadings (e.g., HAQ) could be attributed to differences between study populations (e.g., early vs. established RA, sample size). Apart from traditional clinical and laboratory factors, patient-reported pain, fatigue, functionality and overall well-being determine disease burden, both in early and established RA. Using these factor scores could facilitate detection and management of patient's unmet needs.

\section{REFERENCES:}

[1] Pazmino, et al. Does Including Pain, Fatigue, and Physical Function When Assessing Patients with Early Rheumatoid Arthritis Provide a Comprehensive Picture of Disease Burden? J Rheumatology 2020 Nov.

[2] Berthelot JM, et al. A tool to identify recent or present rheumatoid arthritis flare from both patient and physician perspectives: the 'FLARE' instrument. Annals Rheumatic Diseases. 2012.

Disclosure of Interests: None declared

DOI: 10.1136/annrheumdis-2021-eular.915

\section{AB0110 DETERMINING THE AMOUNT OF COPPER- CONTAINING PROTEIN, ITS BIOCHEMICAL AND IMMUNOLOGIC ACTIVITY IN RHEUMATOID ARTHRITIS PATIENTS}

O. Rusanova ${ }^{1}$, A. Trofimenko ${ }^{1}$, N. Emelyanov ${ }^{2}$, O. Emelyanova ${ }^{1} .{ }^{1}$ Federal State Budgetary Institution «Research Institute of Clinical and Experimental Rheumatology named after A.B. Zborovsky", 1, Волгоград, Russian Federation;

${ }^{2}$ Federal State Budgetary Educational Institution of Higher Education "Volgoorad State Medical University» of the Ministry of Healthcare of the Russian Federation, 2, Волгоград, Russian Federation

Background: Production of antibodies to ceruloplasmin (CP) in rheumatoid arthritis is an issue that has not been studied well enough. It was not by chance that this copper-containing alpha 2-glycorpoteid of blood plasma showing multienzymatic properties was chosen as an object of investigation. Data on the content and activity of $\mathrm{CP}$ in the blood of rheumatoid arthritis patients are contradictory, which has to do with different approaches to selection of patients and different measuring methods.

Objectives: Improving diagnosis of rheumatoid arthritis by determination of antibodies to $\mathrm{CP}$ as well as its amount and enzymatic activity. 
Methods: We studied the serum from 30 apparently healthy individuals, and 108 rheumatoid arthritis patients. Antibodies to CP were determined by enzyme immunoassay using immobilized granulated antigen preparations (modification by Gontar et al, 2002). The amount of CP was determined by enzyme immunoassay according to the method of I.S. Kuzmina et al (1991) using commercial diagnostic agent manufactured by Mechnikov Research Institute for Vaccines and Sera.

Results: Enzyme immunoassay showed a mean level of $\mathrm{CP}$ antibodies in donor sera of $0,020 \pm 0,006$ optical density units. The level of normal values of specific antibodies determined as $\mathrm{M} \pm 2 \sigma$ included an extinction value in the range $0-0,086$. The mean value of oxidase activity and the amount of $C P$ in healthy people was $716 \pm 26,3$ and $921 \pm 32 \mathrm{ng} / \mathrm{ml}$, correspondingly. In the process of study we revealed a reliable increase in CP antibody count, the activity and amount of CP in patients with rheumatoid arthritis while in all cases the parameters under study correlated with the degree of disease activity $(p<0,05)$ : at activity degree I CP antibodies were 0,098 $\pm 0,011$; CP activity was $954 \pm 48,1 ; \mathrm{CP}$ amount was $1292 \pm 73,4$. At activity degree II CP antibodies were $0,138 \pm 0,007$; CP activity was $1163 \pm 39,6$; CP amount was $1763 \pm 69,3$. At activity degree III, CP antibodies were $0,182 \pm 0,015$; CP activity was $1368 \pm 89,5$; CP amount was $1794 \pm 102,8$. After a course of hospital treatment was completed, we noted a reliable decrease in the activity and amount of $\mathrm{CP}$ (at degree I of rheumatoid arthritis activity $\mathrm{p}<0,001$, at degree II of rheumatoid arthritis activity $p<0,01$ for both parameters; at degree III, $p<0,05$ ) compared with baseline findings. A decrease in CP antibodies shows decelerated dynamics, especially in patients with pronounced disease activity, which indicates severe disorders in the immunity that cannot be cured completely within $30-40$ days of hospital treatment course.

Conclusion: Determination of $\mathrm{CP}$ antibodies, as well as quantitative content of $\mathrm{CP}$ and its oxidase activity can serve as indicators of the activity of rheumatoid arthritis, as well as an accessory criterion of the effectiveness of administered therapy.

Disclosure of Interests: None declared

DOI: 10.1136/annrheumdis-2021-eular.948

\begin{tabular}{|l|l}
\hline AB0111 & FAMILIAL RHEUMATOID ARTHRITIS WITH \\
LATE ONSET (50 YEARS AND OLDER) IN \\
CLINICAL PRACTICE ACCORDING TO THE \\
ALL-RUSSIAN ARTHRITIS REGISTER \\
(OREL)
\end{tabular}

A. Satybaldyev ${ }^{1}$, O. Rumyantseva ${ }^{2}$, N. Demidova ${ }^{2}$, E. Gerasimova ${ }^{1}$, E. Strebkova ${ }^{3}$, J. Murtazalieva ${ }^{4}$, E. Koltsova ${ }^{4}$, M. Nikolenko ${ }^{4}$, G. Lukina ${ }^{4}$, E. Zhilyaev ${ }^{4}$, E. Nasonov ${ }^{5} .{ }^{1} V$ A Nasonova Research Institute of Rheumatology, Department of Early Arthritis, Moscow, Russian Federation: ${ }^{2} V$ A Nasonova Research Institute of Rheumatology, Clinical Department, Moscow, Russian Federation; ${ }^{3} V$ A Nasonova Research Institute of Rheumatology, Osteoarthritis Laboratory, Moscow, Russian Federation; ${ }^{4}$ Moscow Research and Practical Center, Moscow Healthcare Department Clinical Moscow, Moscow, Russian Federation; ${ }^{5}$ V A Nasonova Research Institute of Rheumatology, Scientifical Department, Moscow, Russian Federation

Background: Relatives of the 1st degree of relationship (RFDR) of a patient with rheumatoid arthritis (RA) have the higher risk for the development of RA, associated with a 2-5 fold increase.

Objectives: Clinical characteristics of probands with RA onset at an older age ( 50 yrs and older) and their RFDR, which had RA, other rheumatic diseases (RD) and other autoimmune diseases (AID).

Methods: 2766 RA pts with onset of the disease at the age of $50 \mathrm{yrs}$ and older, residents of Moscow and the Moscow region, were included in the Russian register of arthritis OREL in the period from 01.06.2012 to 31.12.2018. 67 probands with RA (2.4\%) had RFDRs with RA, other RDs, other AIDs ( 86 RFDRs in total). Disease activity (by DAS-28), X-ray stage - (by modified Steinbrocker), functional disorders (by functional class - FC), immunological characteristics (RF, ACPA), etc., characterizing the clinical manifestations of RA were evaluated in accordance with the requirements of the current national working RA classification. RFDRs were divided into groups depending on relation to proband father, mother and siblings with RA, RFDRs withr other RD and RFDR with other AID; serological affiliation to RF and ACPA; association with concordant and discordant RFDR, as well as on number of RFDRs ( 1 and 2 or more)

Results: 67 probands with RA were investigated: 9 men, 58 women, average age in debut $(\mathrm{m} \pm \delta) 58.78 \pm 6.23 \mathrm{yrs}$; the average age at the time of the last study was $68.8 \pm 6.38 \mathrm{yrs}$, average duration of RA was $10.14 \pm 5.69 \mathrm{yrs} .6(9 \%)$ probands had FC1, 45 (69\%) - FC2, 13 (20\%) - FC3, 1 (2\%) - FC4, 2 - without data; $34(53 \%)$ had high activity, $23(36 \%)$-medium, $6(9 \%)$ - minimal, $1(2 \%)$ remission, 3 without data; RF-positive (RF+) - 77.6\%, ACPA-positive (ACPA+) $71.7 \% .19$ probands (32\%) had 1-2a X-ray stage, $27(46 \%)$ - stage 2 b, $10(17 \%)$ - stage 3, $3(5 \%)$ - stage 4, 8 - without data. $61(91 \%)$ had advanced clinical stage, $6(9 \%)$ - late. Among 86 RFDRs (67 - RF+, 19 - RF-) 10 had father with RA (8 RF+ probands, 2 - RF-), 2 - father with other RDs (all RF+), 10 - mother with RA (all RF+), 7 - mother with other RDs (4 - RF+, 3 - RF-), 26 - siblings with RA (20 - RF+, 6 - RF-), 11 - siblings with other RDs (9 - RF+, 2 - RF-), 20 - RFDR with other AIDs (14 - RF+, 6 - RF-). Probands with concordant RFDRs had more pronounced changes: high activity ( $56 \%$ versus $32 \%)$, X-ray erosive stages $(67 \%$ versus $60 \%)$ and stages III and IV (32\% versus $6 \%)$; FC3 and FC4 $(23 \%$ versus $12 \%)$ and a tendency towards more frequent development of the late clinical stage ( $11 \%$ versus $2 \%)$. Comparison of clinical signs in probands with 1 RFDR (49 cases) and probands with 2 or more RFDRs (17 cases) showed a significant predominance of high inflammatory activity $(61 \%$ versus $41 \%$ ), erosive arthritis ( $77 \%$ versus $57 \%$ ), and a tendency to development of a late clinical stage (10\% versus $6 \%$ ) in probands with 1 RFDR. In probands with RF-positive RA, the predominance becomes more significant: high inflammatory activity (69\% versus $43 \%$ ), erosive arthritis ( $90 \%$ versus $54 \%$ ), late clinical stage ( $13 \%$ versus $7 \%$ ), and a tendency to severe functional impairment - FC3 and FC4 (22\% vs. 15\%). ACPA-positive probands showed the same tendency as RF-positive.

Conclusion: The clinical picture of probands with RFDRs with RA, other RDs, other AIDs is generally characterized by more pronounced changes in probands with RA who have RFDRs with concordant diseases in the form of more often high inflammatory activity, less often moderate, erosive arthritis, more frequent development of advanced 3 and $4 \mathrm{X}$-ray stages (every fifth). Also, more pronounced changes develop in probands with RA who have only 1 RFDR compared to probands with 2 or more RPSRs. In this group, the differences are even more pronounced in the case of RF-positive probands. In cases with ACPA-positive probands, the same trends persist. Careful interview of a proband with RA about the presence of RFDRs with RA, other RDs and other AIDs will make it possible to predict its course more correctly and facilitate planning of rational therapy.

Disclosure of Interests: None declared

DOI: 10.1136/annrheumdis-2021-eular.962

\section{$\mathrm{AB} 0112$ \\ TNF INHIBITOR MONOTHERAPY IN RHEUMATOID ARTHRITIS: IS THERE REALLY A DIFFERENCE IN COMPARISON WITH COMBINATION THERAPY WITH CSDMARDS IN REAL-LIFE?}

B. M. Fernandes ${ }^{1}$, S. Garcia ${ }^{1}$, F. Oliveira Pinheiro ${ }^{1}$, M. Rato ${ }^{1}$, D. Fonseca ${ }^{2}$, D. Santos Oliveira ${ }^{1,3}$, A. Martins ${ }^{1}$, F. R. Martins ${ }^{4}$, M. Bernardes ${ }^{1,5}$, L. Costa ${ }^{1}$ ${ }^{1}$ Centro Hospitalar Universitário São João, Rheumatology, Porto, Portugal; ${ }^{2}$ Centro Hospitalar Vila Nova de Gaia/Espinho, Rheumatology, Vila Nova de Gaia, Portugal; ${ }^{3}$ Faculty of Medicine, University of Porto, Center for Health Technology and Services Research (CINTESIS), Porto, Portugal; ${ }^{4}$ Centro Hospitalar Universitário do Algarve, Rheumatology, Faro, Portugal; ${ }^{5}$ Faculty of Medicine, University of Porto, Departamento de Medicina, Porto, Portugal

Background: In Rheumatoid Arthritis (RA), tumor necrosis factor inhibitors (TNFi) in combination with conventional synthetic disease-modifying antirheumatic drugs (csDMARDs) has shown advantages concerning efficacy and immunogenicity in comparison with monotherapy. However, in clinical practice, up to $40 \%$ of patients under biological DMARDs (bDMARDs) are on monotherapy. Objectives: To compare the efficacy outcomes of TNFi in monotherapy and in combination therapy in a RA monocentric cohort.

Methods: Retrospective, cross-sectional study including all the RA patients under TNFi followed at our Rheumatology Department and registered in the national database. Demographic, clinical and laboratorial data and disease activity measures were collected at the last visit of 2019 from each patient. Mann-Whitney $\mathrm{U}$ and chi-square tests were used to the comparison analysis between the two groups (continuous and categorical variables, respectively).

Results: A total of 144 patients were included: $84 \%$ were females; at the last visit of 2019 , the mean age was $56.3 \pm 10.9$ years and the mean disease duration was $18.3 \pm 10.2$ years; $73.6 \%$ were positive for rheumatoid factor (RF), $81.9 \%$ for anti-citrullinated protein autoantibodies (ACPA) and $45.1 \%$ had erosive disease. There were no statistically significant differences in these variables between the monotherapy and the combination therapy groups (table 1). 